\title{
Structure-Property Relationships in Aligned Electrospun Barium Titanate
} Nanofibers

\author{
Matthew J. Bauer, ${ }^{\star}$ Catherine S. Snyder, ${ }^{\star}$ Christopher C. Bowland, ${ }^{\ddagger}$ Amanda M. Uhl, ${ }^{\star}$ Maeve A. K. \\ Budi, ${ }^{\ddagger}$ Maria Villancio-Wolter, ${ }^{\ddagger}$ Henry A. Sodano, ${ }^{\S \uparrow}$ and Jennifer S. Andrew ${ }^{\ddagger} \dagger$ \\ tDepartment of Materials Science and Engineering, University of Florida, Gainesville, Florida 32611-6400 \\ ${ }^{\S}$ Department of Aerospace Engineering, University of Michigan, Ann Arbor, Michigan 48109-2140 \\ "Department of Materials Science and Engineering, University of Michigan, Ann Arbor, Michigan 48109
}

\begin{abstract}
Barium titanate nanofibers were uniaxially aligned by electrospinning onto a rotating copper wire drum and alignment was maintained during calcination of the fibers. Two methods for maintaining alignment during calcination were tested, by either using carbon tape or a peeling off method to remove the aligned fibers from the mandrel followed by calcination. The carbon tape removal method led to the formation of shorter aligned nanowires while the peeling off method resulted in longer nanofibers. Additionally, the effects of calcination temperature and time on crystal structure were also examined. The degree of tetragonality in the barium titanate nanofibers increased at higher calcination temperatures and times. Piezoelectricity was confirmed in the nanofibers calcined using piezoeresponse force microscopy, yielding a $d_{33}$ value of $15.5 \mathrm{pm} / \mathrm{V}$. Using the methods presented here, large quantities of aligned piezoelectric barium titanate and other ceramic fibers or wires can be produced to fulfill their demand in novel microelectronics.
\end{abstract}

Keywords: barium titanate; electrospinning; sol-gel

\section{Introduction}

$\mathrm{E}$ LECTROSPINNING is a versatile method for producing nano- to micrometer sized fibers that are suitable for both rapid prototyping and large-scale production. ${ }^{1}$ Electrospinning has been used to synthesize a wide array of nanoscale materials including polymeric, ${ }^{2-4}$ ceramic, ${ }^{5-8}$ and even composite bi- and multiphasic fibers and particles. ${ }^{9-12}$ Fibers formed via eletrospinning are promising candidates for use in many applications including filtration, catalysis, wound healing, and drug transport and release. ${ }^{1}$ Traditionally, electrospinning results in the formation of randomly oriented fibers. However, fiber alignment can be achieved by tailoring the geometry of the counter electrode. ${ }^{4,13}$ In some applications, electrospinning a large mat of randomly oriented fibers is sufficient, whereas for others, alignment or a desired orientation in the electrospun fibers may be desired. ${ }^{4,13,14}$ This paper demonstrates a route to produce large quantities of uniaxially aligned barium titanate nanofibers in a manner that can also be applied to a wider range of ceramic and metallic electrospun nanofibers.

The piezoelectric and ferroelectric properties and high dielectric constant of barium titanate have enabled its incorporation into a wide array of electrical applications including actuators, ${ }^{15}$ medical imaging devices, ${ }^{16}$ ultrasound transducers, ${ }^{17}$ and high dielectric capacitors. ${ }^{18}$ Nanoscale barium titanate has been considered a promising material for high-density ferroelectric random access memories. ${ }^{14,19-22}$

Manuscript No. 38239. Received February 25, 2016; approved July 16, 2016.

${ }^{\dagger}$ Author to whom correspondence author should be addressed. e-mail: jandrew@mse.ufl.edu
Additionally, electrospun barium titanate nanofibers have been used to construct high-performance humidity sensors in place of barium titanate thin films due to their high surface area and large aspect ratio. ${ }^{21,22}$ A key figure of merit for barium titanate nanowires is their piezoelectric coefficient, $d$, which is a measure of the magnitude of polarization that is generated in response to an applied load. In particular, the $d_{33}$ piezoelectric coefficient is often reported, which measures the piezoelectric response along the axis of the applied electric field.

Given the push toward incorporating barium titanate and similar perovskite nanofibers and nanowires into electrical devices, it is important to develop methods to synthesize large quantities of these nanofibers. ${ }^{14,19,20}$ Control of their orientation is desirable since randomly oriented fibers may not be best suited for use in microelectronics. While wellaligned barium titanate/polymer nanofibers have been previously synthesized with the use of two parallel electrodes separated by an insulating air gap, ${ }^{14,20}$ the area of fibers which can be produced with this method is limited by the distance between the electrodes and their length. The method is further limited by a loss in alignment as subsequent fiber layers are deposited. ${ }^{4}$ This has been overcome in polymer systems by parallel electrodes spaced around a rotating mandrel. ${ }^{4}$ However, for ceramic nanofibers compared to polymer fibers, there is an additional challenge of maintaining alignment during calcination. The as-spun fibers are amorphous; thus, a calcination step is required to burn off the polymer binder and to form the final crystalline product.

This paper demonstrates the first example of alignment in ceramic nanofibers that persists during calcination by electrospinning onto a rotating mandrel. In this paper, two methods of maintaining fiber alignment throughout the calcination process are explored. The first method involves the removal of the as-spun fibers using carbon tape and the other involves the removal of sections of fibers by peeling them off the wire drum as sheets. In this work, we also examine the effects of other processing variables, including calcining time and temperature on the structure and properties of the resultant nanofibers.

\section{Experimental Process}

\section{(1) Materials}

Barium acetate, poly(vinyl pyrrolidone) (PVP, $\mathrm{MW}=1$ $300000)$, acetic acid, and titanium isopropoxide $(\geq 97.0 \%)$ were obtained from Sigma Aldrich (St. Louis, MO).

\section{(2) Barium Titanate Sol-Gel Preparation}

The barium titanate precursor solution was prepared in a 1:1 molar ratio of barium to titanium by first dissolving $2.55 \mathrm{~g}$ 
barium acetate in $8 \mathrm{~mL}$ of acetic acid followed after $2 \mathrm{~h}$ by the dropwise addition of $2.95 \mathrm{~mL}$ titanium isopropoxide. ${ }^{11,19,23}$ A polymer solution was simultaneously prepared, consisting of $1.1 \mathrm{~g}$ PVP $(\mathrm{MW}=1300000)$ in $10 \mathrm{~mL}$ ethanol and was also stirred for $2 \mathrm{~h}$. Then, the barium titanate solution was added to the polymer solution and allowed to stir for $10 \mathrm{~min}$ before electrospinning.

\section{(3) Electrospinning Aligned Barium Titanate Fibers}

To electrospin aligned nanofibers, a counter electrode consisting of parallel copper wires was constructed on a rotating

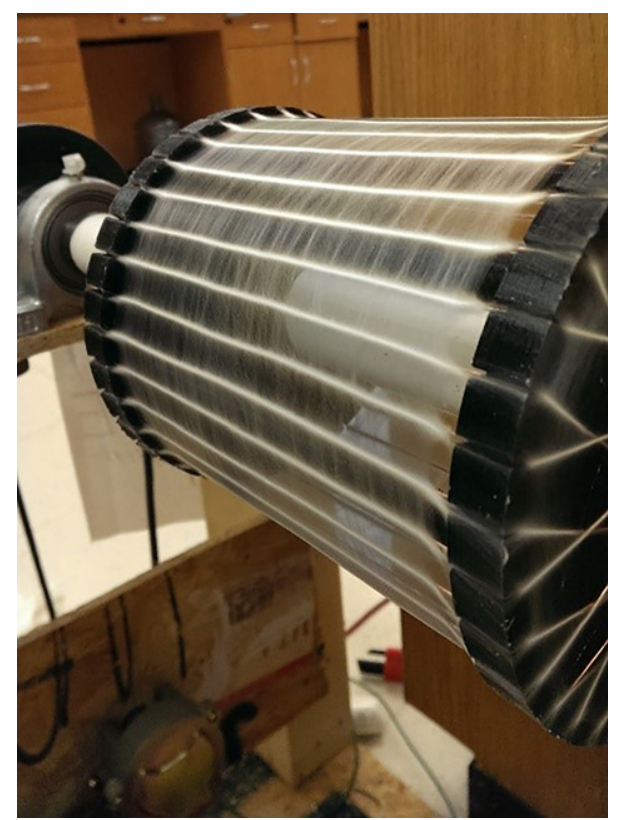

Fig. 1. Photograph of the rotating mandrel set-up for the electrospinning of aligned nanofibers showing the as-spun amorphous fibers aligned across the parallel copper wires on the rotating mandrel. mandrel (Fig. 1). The copper wires had a separation of $1 \mathrm{~cm}$ and the drum was rotated by a DC electric motor at a rate of $21 \mathrm{rpm}$, determined by the rotational speed of the DC motor and the pulley diameters.

For electrospinning, the barium titanate precursor solution was fed through a stainless steel needle ( 20 gage) with a flow rate of $2 \mathrm{~mL} / \mathrm{h}$. The needle was connected to a high voltage power supply, with an applied voltage of $13 \mathrm{kV}$, and positioned normal to the surface of the electrically grounded rotating copper wire drum, with a separation distance of $16 \mathrm{~cm}$. After electrospinning, two methods were tested for the removal of as-spun fibers from the copper wire mandrel for calcination: removal by peeling the fibers off as sheets or by adhering the aligned fibers to a piece of carbon tape. The as-spun fibers were calcined at $750^{\circ} \mathrm{C}, 875^{\circ} \mathrm{C}$, and $1000^{\circ} \mathrm{C}$ for 2,4 , or $6 \mathrm{~h}$.

\section{(4) Characterization}

The viscosity of five barium titanate precursor solutions was measured using a Brookfield DV-II+ Pro rotational viscometer with a spindle speed of $20 \mathrm{rpm}$. The average viscosity of the as-prepared precursor solutions was $137 \mathrm{cP}$ with a standard deviation of $14 \mathrm{cP}$.

The nanofiber samples were imaged with a FEI Phillips (Hillsboro, OR) XL40 FEG scanning electron microscope (SEM). Fiber diameters were measured using ImageJ $(\mathrm{NIH}$, Bethesda, MD), where diameter measurements were taken on at least 150 fibers per sample. The measured fiber diameters were then fit to multiple normal distributions using normal mixEM $^{24}$ in Rstudio (Boston, MA). A fit for multiple log normal distributions was found by first taking the log transform of the diameter measurements, fitting the data with normal mixEM, and converting the normal fits of the log transformed data back into log normal distributions. The crystal structure of the nanofibers was determined via X-ray diffraction (XRD) (PANalytical X'Pert Powder, Alemelo, the Netherlands) with subsequent Rietveld refinement in PANalytical Highscore Plus. Additional crystallographic structure information was obtained using Raman spectroscopy; Raman spectra were obtained using a Renishaw Invia Raman
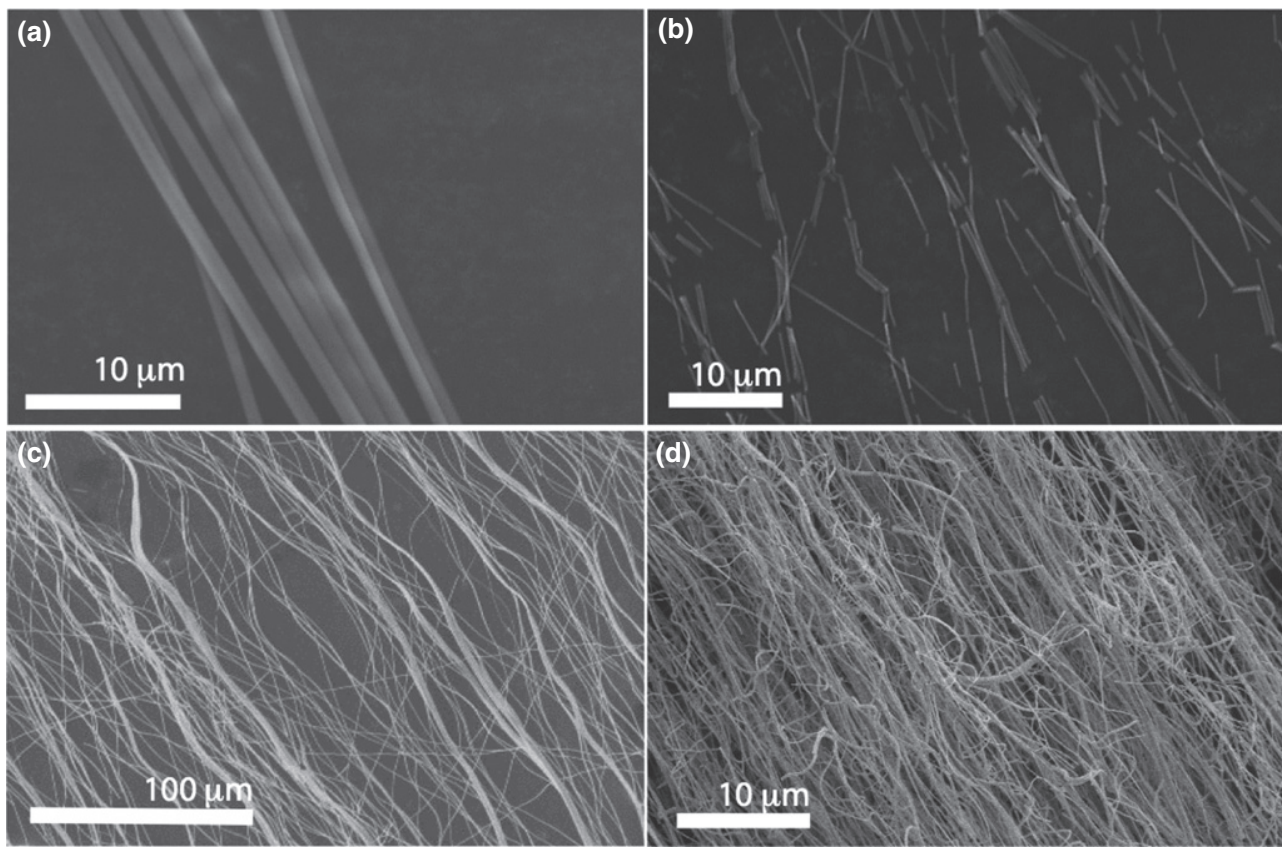

Fig. 2. Scanning electron micrographs of (a) uncalcined, aligned nanofibers (b) aligned barium titanate nanofibers, calcined at $750^{\circ} \mathrm{C}$ for $2 \mathrm{~h}$ after removal via the carbon tape method, and aligned nanofibers calcined at (c) $750^{\circ} \mathrm{C}$ for $2 \mathrm{~h}$ and (d) $1000^{\circ} \mathrm{C}$ for $2 \mathrm{~h}$ after removal by the peeling off method. 
microscope (Hoffman Estates, IL) with a $633 \mathrm{~nm}$ laser and a $20 \times$ objective lens.

Piezoelectric force microscopy (PFM) was performed using a Park Systems XE 70 atomic force microscope (Santa Clara, CA) modified with a function generator (Agilent, 33210A, Santa Rosa, CA). PFM samples were prepared by electrospinning directly onto platinum-coated silicon wafers with a layer of titanium dioxide between the platinum and silicon to promote adhesion of the thin film, after which they were calcined for $2 \mathrm{~h}$ at $750^{\circ} \mathrm{C}$. The $\mathrm{d}_{33}$ value of a barium titanate nanofiber calcined at $750^{\circ} \mathrm{C}$ for $2 \mathrm{~h}$ was obtained via linear regression on the positive voltage sweep using 20 cycles.

\section{Results and Discussion}

After electrospinning onto the copper wire drum, SEM images were obtained to verify alignment and to observe the morphology of the as-spun fibers [Fig. 2(a)]. After the alignment of the as-spun fibers was verified, the fibers were removed from the copper wire mandrel using two different methods. In the first method, fibers were removed by adhering them to carbon tape. The carbon tape was then placed on a ceramic dish, and was calcined in air at $750^{\circ} \mathrm{C}$ for $2 \mathrm{~h}$, resulting in the oxidation and removal of the carbon tape from the fibers [Fig. 2(b)]. The adhesive on the carbon tape restricted the shrinking of the fiber as the PVP was removed, leading to the fibers being pulled apart lengthwise, breaking to form aligned nanowires instead of longer nanofibers [Figs. 2(b-c)].

The second method of maintaining alignment during calcination involved removing the fibers by peeling off sections of sheets from the copper wire drum. Using this method, the fibers were free to shrink along their length during calcination. Figures $2(\mathrm{c}-\mathrm{d})$ show that this sheet removal method maintained alignment and resulted in uniaxially aligned nanofibers, after calcination at $750^{\circ} \mathrm{C}$ and $1000^{\circ} \mathrm{C}$, respectively.

Figures 3(a) and (b) show the fiber diameters distributions for both calcined and uncalcined fibers, respectively, revealing a bimodal distribution of fiber diameters. This multimodal distribution has been previously observed in both polymer $^{25,26}$ and sol-gel electrospinning ${ }^{27}$ and has been attributed to branching, the formation of secondary polymer/ sol-gel jets from the primary jet, during the electrospinning process. This branching phenomena is due to an instability of the circular cross section of the fiber at high potentials. This instability causes the formation of cusps from which secondary jets can be emitted. ${ }^{25}$ Though previously such multimodal fiber distributions have been modeled using multiple normal distributions, ${ }^{26}$ we found that the diameters of the electrospun fibers were better fit by multiple log normal distributions. The origin of this log normal fiber diameter distribution can be related to solution inhomogeneities that are present during electrospinning. In electrospinning, a polymer or sol-gel solution is placed in a syringe that is connected to a high voltage power supply, which leads to the formation of an elongated droplet at the syringe tip, referred to as the Taylor Cone. The radius of the Taylor cone has been shown to be a determining factor of the droplet size in electrospraying, ${ }^{28}$ and it has also been predicted that it is related to the final jet diameter for electrospinning. ${ }^{29}$ Additionally, the radius of the Taylor cone is proportional to the viscosity of the fluid, ${ }^{30}$ where the viscosity of the polymer is related exponentially to its concentration, as described by the Martin equation, which varies randomly throughout the solution. ${ }^{31}$ These random variations then give rise to a viscosity that is $\log$ normally distributed, resulting in a Taylor cone radius and fiber diameters that also exhibit a log normal distribution (Fig. 3).

The fiber diameter distributions from both normal and $\log$ normal models were graphically compared to the
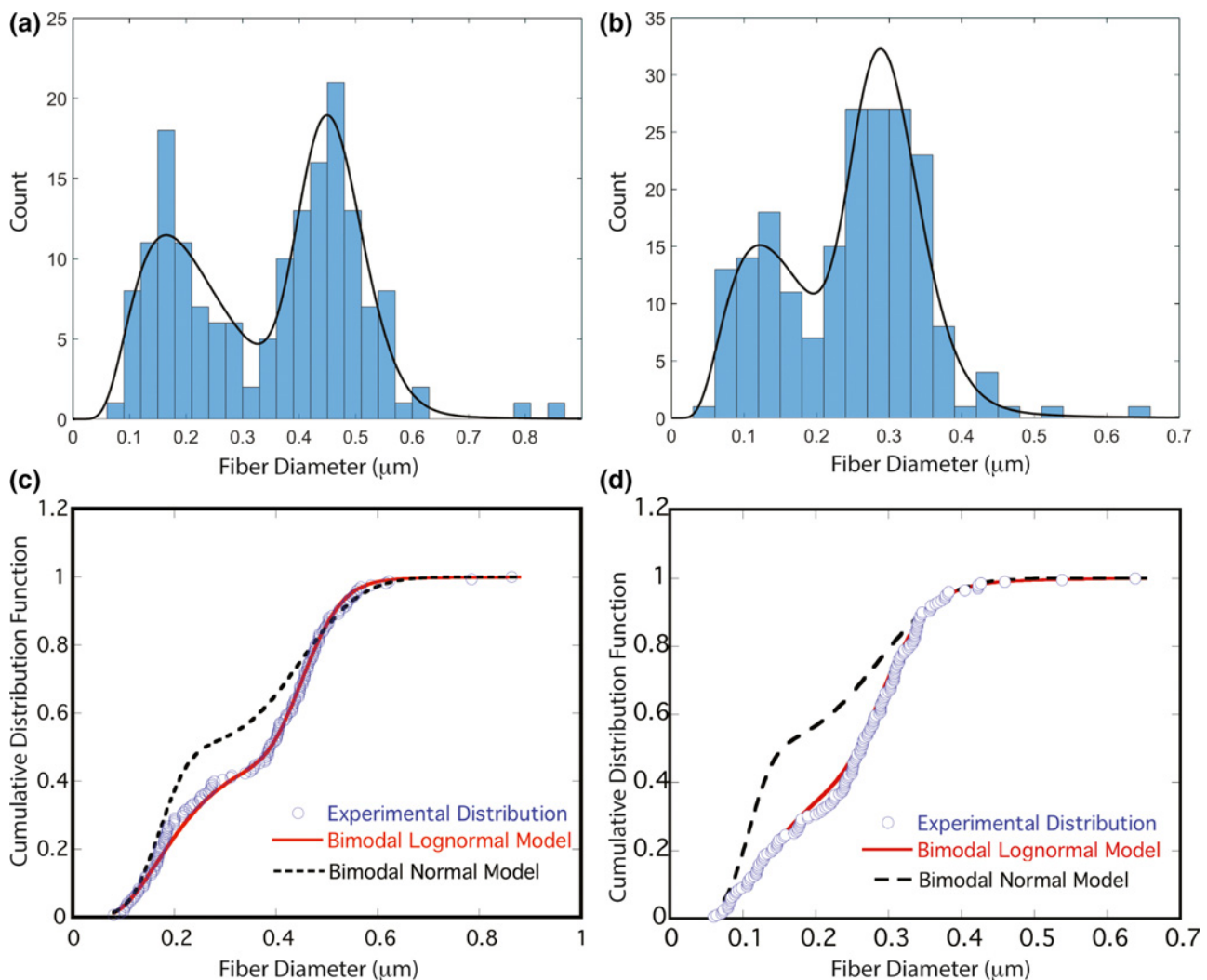

Fig. 3. Fiber diameter distributions for (a) uncalcined barium titanate fibers and (b) calcined barium titanate fibers with the log normal probability density function given by the black line. Experimental cumulative distribution of the (c) uncalcined and (d) calcined barium titanate fibers and along with fitted bimodal normal and log normal distributions. 

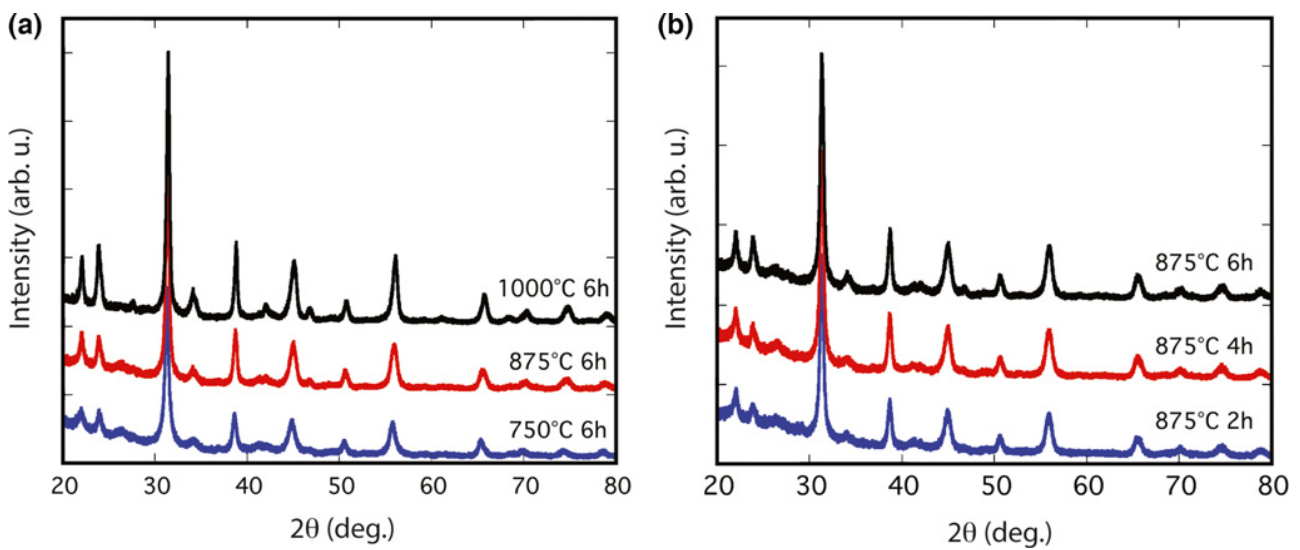

Fig. 4. (a) X-ray diffraction (XRD) spectra for barium titanate nanofibers calcined for $6 \mathrm{~h}$ at $750^{\circ} \mathrm{C}, 875^{\circ} \mathrm{C}$, and $1000^{\circ} \mathrm{C}$. (b) XRD spectra for barium titanate nanofibers calcined at $875^{\circ} \mathrm{C}$ for 2,4 , and $6 \mathrm{~h}$.
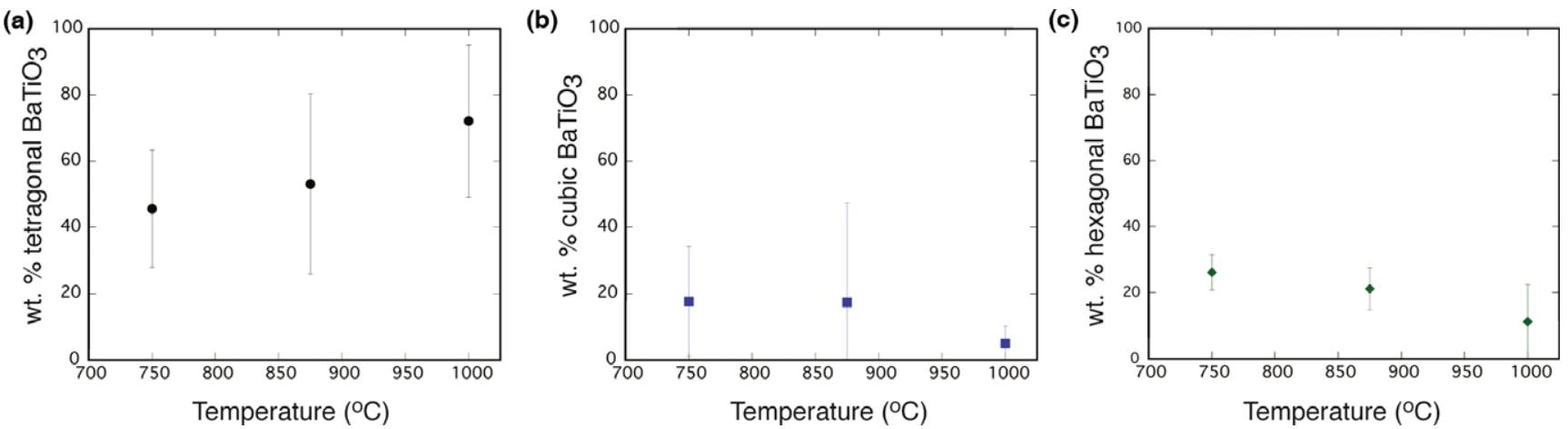

Fig. 5. The weight percent of the (a) tetragonal, (b) cubic, and (c) hexagonal phase of barium titanate as a function of calcining temperature, for a constant calcining time of $6 \mathrm{~h}$.

experimental cumulative distribution [Figs. 3(c-d)]. The fit of each model distribution was also quantitatively evaluated using the Kolmogorov-Smirnov $(\mathrm{K}-\mathrm{S})$ test to determine if the model distribution is a reasonable fit for the experimental results. ${ }^{32}$ Based on the $\mathrm{K}-\mathrm{S}$ test, the multiple normal distributions could be rejected for both the uncalcined $\left(P=1.60 \times 10^{-4}\right)$ and calcined $\left(P=2.60 \times 10^{-14}\right)$ fibers. The multiple log normal distributions could not be rejected with the K-S test with a $95 \%$ confidence level, thus the multimodal log normal distribution model was accepted. The multiple log normal distribution for the uncalcined fibers can be defined as follows: the first distribution had a weight of 0.58 , a mean diameter of $232 \pm 117 \mathrm{~nm}$, while the second had a weight of 0.42 , a mean of diameter of $463 \pm 58 \mathrm{~nm}$. For the calcined multiple log normal distribution, the first distribution had a weight of 0.447 , a mean diameter of $180 \pm 98 \mathrm{~nm}$, while the second had a weight of 0.553 , a mean diameter of $302 \pm 47 \mathrm{~nm}$.

The crystallographic properties of the calcined nanofibers were analyzed using XRD. The XRD spectra are shown in Fig. 4, revealing peaks characteristic of perovskite barium titanate. ${ }^{14,19,33}$ Rietveld refinement indicated the presence of the tetragonal perovskite structure for all calcination conditions. However, Fig. 4 shows a lack of observable peak splitting in the (200) peak at $45^{\circ}$, which can be attributed to peak broadening in the nanocrystalline samples. ${ }^{33-35}$ The Rietveld refinement also indicated the presence of a cubic and hexagonal phase of barium titanate, in addition to the presence of barium carbonate (witherite). The metastable hexagonal phase has been previously shown to coexist with the tetragonal phase of barium titanate in nanoparticles. The phase transformation between the hexagonal and tetragonal phases is a reconstructive phase transformation, and thus characterized by sluggish kinetics. ${ }^{36}$ Therefore, as anticipated, the amount of hexagonal phase present in the sample decreases
Table I. Agreement Indices for the Rietveld Refinements on the Barium Titanate Fibers

\begin{tabular}{|c|c|c|c|c|c|}
\hline Temp $\left({ }^{\circ} \mathrm{C}\right)$ & Time (h) & Trial & $R_{\exp }(\%)$ & $R_{\mathrm{wt}}(\%)$ & $\chi_{\mathrm{v}}^{2}$ \\
\hline \multirow[t]{3}{*}{750} & 2 & 1 & 5.03 & 5.99 & 1.42 \\
\hline & & 2 & 6.12 & 6.50 & 1.13 \\
\hline & & 3 & 6.49 & 7.08 & 1.19 \\
\hline \multirow[t]{3}{*}{750} & 4 & 1 & 6.42 & 7.95 & 1.54 \\
\hline & & 2 & 5.76 & 6.13 & 1.13 \\
\hline & & 3 & 6.87 & 7.45 & 1.18 \\
\hline \multirow[t]{3}{*}{750} & 6 & 1 & 4.89 & 5.05 & 1.07 \\
\hline & & 2 & 6.22 & 7.52 & 1.46 \\
\hline & & 3 & 6.64 & 7.56 & 1.30 \\
\hline \multirow[t]{3}{*}{875} & 2 & 1 & 5.09 & 6.00 & 1.39 \\
\hline & & 2 & 5.88 & 6.22 & 1.12 \\
\hline & & 3 & 6.60 & 7.16 & 1.18 \\
\hline \multirow[t]{3}{*}{875} & 4 & 1 & 4.95 & 6.19 & 1.57 \\
\hline & & 2 & 6.14 & 7.08 & 1.33 \\
\hline & & 3 & 6.75 & 7.44 & 1.21 \\
\hline \multirow[t]{3}{*}{875} & 6 & 1 & 5.13 & 5.88 & 1.31 \\
\hline & & 2 & 6.29 & 7.36 & 1.37 \\
\hline & & 3 & 6.61 & 7.03 & 1.13 \\
\hline \multirow[t]{3}{*}{1000} & 2 & 1 & 6.52 & 8.47 & 1.69 \\
\hline & & 2 & 6.65 & 8.34 & 1.57 \\
\hline & & 3 & 6.86 & 7.68 & 1.25 \\
\hline \multirow{3}{*}{1000} & 4 & 1 & 4.92 & 6.34 & 1.66 \\
\hline & & 2 & 6.31 & 7.28 & 1.33 \\
\hline & & 3 & 6.69 & 7.69 & 1.32 \\
\hline \multirow[t]{3}{*}{1000} & 6 & 1 & 6.42 & 7.95 & 1.54 \\
\hline & & 2 & 6.54 & 7.94 & 1.48 \\
\hline & & 3 & 6.82 & 8.97 & 1.73 \\
\hline
\end{tabular}

Agreement indices for the Rietveld refinements performed. $R_{\mathrm{wt}}$ and $R_{\exp }$ are the weighted and expected $R$-factors, respectively and $\chi_{\mathrm{v}}^{2}$ is the reduced chi-square statistic. 
(a)

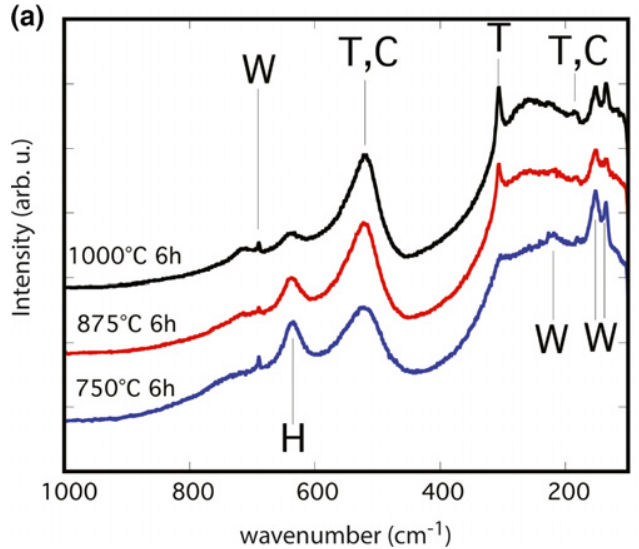

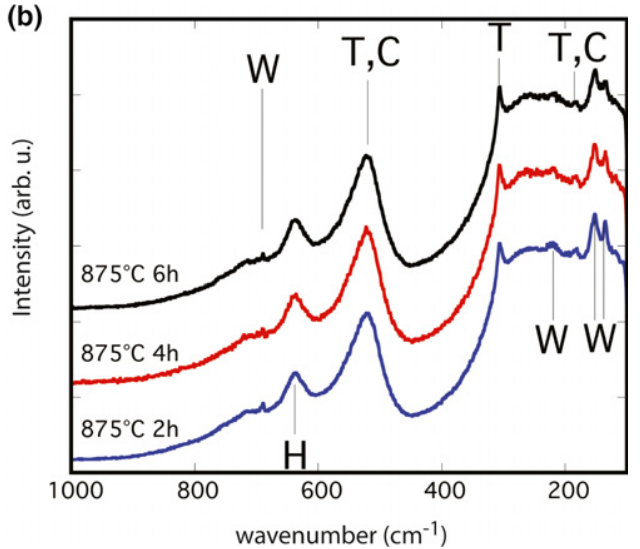

Fig. 6. (a) Raman spectra for barium titanate nanofibers calcined for $6 \mathrm{~h}$ at $750^{\circ} \mathrm{C}, 875^{\circ} \mathrm{C}$, and $1000^{\circ} \mathrm{C}$ and (b) Raman spectra for barium titanate nanofibers calcined at $875^{\circ} \mathrm{C}$ for 2,4 , and $6 \mathrm{~h}$. Peaks are labeled as being attributed to tetragonal barium titanate $(T)$, cubic barium titanate $(C)$, hexagonal barium titanate $(H)$, or barium carbonate (witherite, $W$ ).

with increasing calcination temperature, along with a corresponding increase in the amount of tetragonal phase present, when calcination time is kept constant (Fig. 5). It is important to note, that all samples had a barium carbonate impurity phase, which varied from $10-14 \mathrm{wt} \%$ depending on the calcination temperature. Across all calcination times, temperature was negatively $(R=-0.621)$ and significantly $(P=0.0005)$ correlated with the $\mathrm{wt} \%$ of the intermediate phase as more of the recombination transformation occurred. Furthermore, the percent of the tetragonal phase was positively $(R=0.522)$ and significantly $(P=0.005)$ correlated with increasing calcination temperature and attributed to the more complete transformation of the metastable hexagonal phase at higher temperatures, where more energy was available to promote the kinetically sluggish transformation. Similarly, longer calcination times also corresponded to an increasing $\mathrm{wt} \%$ of the tetragonal phase, and a decrease in the amount of hexagonal phase present. However, we observed that calcination time has a lesser impact on the phases present compared with temperature for the temperatures and times tested. Lastly, both cubic barium titanate and barium carbonate decreased in weight percent with increasing calcination times and temperatures. Rietveld refinement results are given in Table I, where three samples were synthesized and analyzed under each calcination condition. $R_{\mathrm{wp}}$ is a measurement of the distance between the observed and fitted spectra, $R_{\exp }$ is a measure of the data quality and essentially the expected $R_{\text {up }}$ for an ideal fit, and the reduced chi-square statistic $\chi_{\mathrm{v}}^{2}=\frac{R_{\mathrm{wt}}}{R^{2}}$ gives an indication of the quality of the Rietveld refinement on the spectra. For example, a $\chi_{V}^{2}<1$ indicates the spectra may be over fitted, $\chi_{\mathrm{v}}^{2} \gg 1$ indicates a poor fit, and $\chi_{\mathrm{v}}^{2} \approx 1$ signifies a good fit.

Raman spectroscopy was also employed to further confirm the presence and transformation between the metastable hexagonal and tetragonal phases of barium titanate. Figures 6(a) and (b) show the evolution of normalized Raman spectra as a function of calcination temperature and time, respectively. Peaks that are attributed to tetragonal $(T),{ }^{36-39}$ cubic $(C),{ }^{40-42}$ and hexagonal $(H)^{36,37,43}$ barium titanate as well as barium carbonate (witherite, $W)^{43}$ are labeled within the spectra. Figure 6(a) reveals that the tetragonal $(T)$ peak intensities increase with increasing temperature, alongside a corresponding decrease in the intensities of the metastable hexagonal $(H)$ phase. However, Figure 6(b) shows the evolution of Raman spectra at calcination times of 2, 4, and $6 \mathrm{~h}$, for a constant calcination temperature of $875^{\circ} \mathrm{C}$. It is apparent here that the calcination temperature has a greater effect on the transformation from the hexagonal to the tetragonal phase, as also observed in the XRD data.

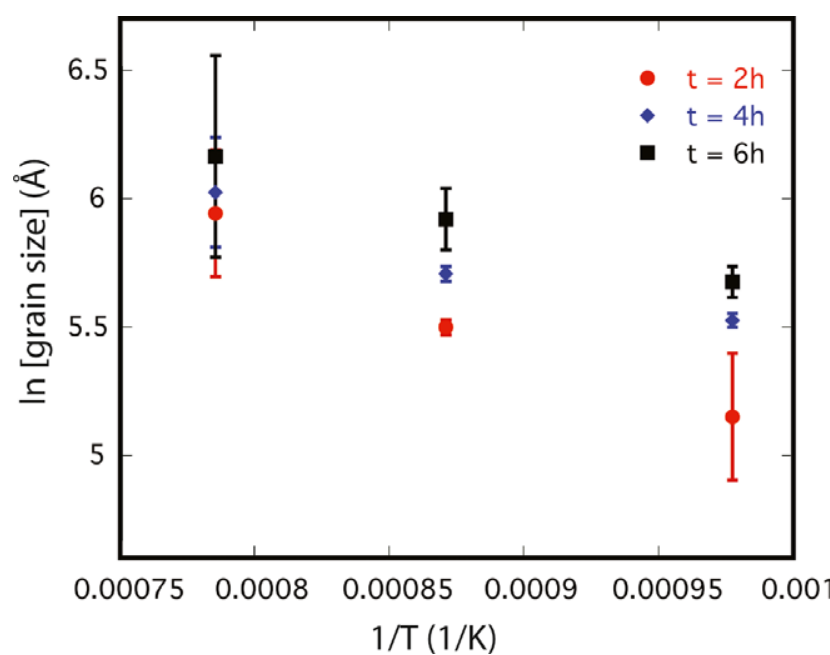

Fig. 7. The natural logarithm of average grain size plotted versus reciprocal temperature for the tetragonal phase. The slope from these plots was used to calculate the activation energy for grain growth in the barium titanate nanofibers.

It is anticipated that barium titanate nanofibers with the highest fraction of the tetragonal phase will result in the best ferroelectric and piezoelectric properties. Therefore, the effects of calcination on the grain size of the tetragonal barium titanate phase were also determined by relating peak broadening to the average grain size. Since strain can also contribute to peak broadening, strain was also considered in the Rietveld refinement, however, no significant strain contributions to peak broadening were found. Figure 7 shows a plot of the natural logarithm of grain size of the barium titanate nanofibers as a function of inverse temperature. From Fig. 7, the activation energy of grain growth of tetragonal barium titanate was calculated to be $2.641 \pm .377 \mathrm{eV} /$ (atom $K)$. This fits in to a trend previously observed in barium titanate nanofibers by Peng et al. where the activation energy of grain growth was found to decrease with decreasing fiber diameter. $^{44}$

Lastly, the piezoelectric properties of the electrospun barium titanate nanofibers were determined using PFM. Figure 8(a) shows the butterfly loop, with corresponding phase plot Fig. 8(b) demonstrating a $180^{\circ}$ phase shift, obtained from PFM analysis on a barium titanate nanofiber calcined at $750^{\circ} \mathrm{C}$ for $2 \mathrm{~h}$, confirming piezoelectric behavior. The $d_{33}$ value, extracted via linear regression on Fig. 8(a) was found to be $15.5 \mathrm{pm} / \mathrm{V}$ with a standard deviation of $0.1 \mathrm{pm} / \mathrm{V}$, in a method established by Zhou et al. ${ }^{45}$ The standard deviation 
(a)

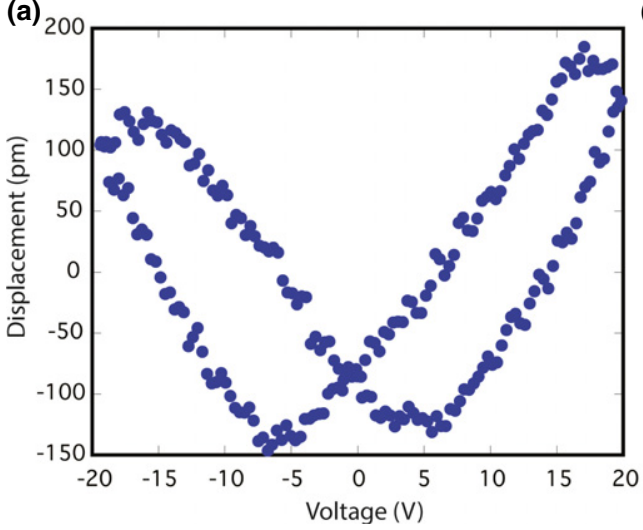

(b) 1

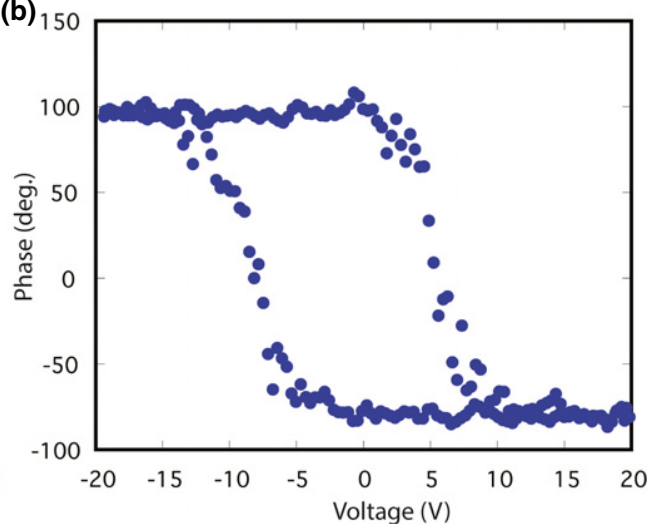

Fig. 8. (a) Butterfly loop and (b) phase plot of barium titanate nanofiber calcined at $750^{\circ} \mathrm{C}$ for $2 \mathrm{~h}$.

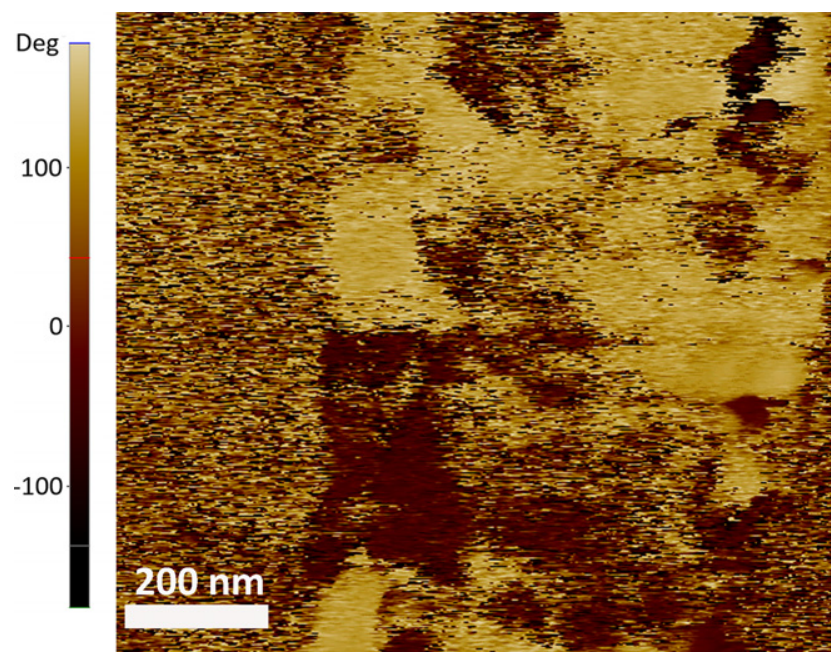

Fig. 9. Phase angle scan of a barium titanate nanofiber calcined at $750^{\circ} \mathrm{C}$ for $2 \mathrm{~h}$, revealing the domain structure of the fiber, the width of the scan shown is $1 \mu \mathrm{m}$.

reported here refers to that of the fitting parameter based on the 20 repeated scans used to obtain the butterfly loop that is shown in Fig. 8(a). The value reported here is also in good agreement, with a $20 \mathrm{pm} / \mathrm{V}$ value previously reported for piezoelectric barium titanate nanofibers. ${ }^{46}$ However, it is important to note that there are several biases present in PFM measurements, including the effect of surface charges which act upon the body of the PFM cantilever, in addition to the forces that are acting solely on the tip. ${ }^{47}$ For cases where domains are more randomly oriented and relatively small in comparison to the cantilever dimensions, this bias is reduced, if not eliminated. ${ }^{47}$ Figure 9 shows the phase angle scan of a barium titanate nanowire, revealing that the as-calcined nanofibers exhibit a random distribution of domains, that are much larger than the cantilever tip. It is anticipated that all calcination conditions would result in the formation of a random domain structure, requiring subsequent poling steps. Although one-dimensional piezoelectrics cannot easily be poled using conventional techniques, methods have been developed where arrays of piezoelectric nanowires are assembled across electrodes, whereby electrical contacts can be made across both ends of the nanowire so as to apply a voltage along the length of the wire. ${ }^{48}$ Additionally, a corona discharge poling have been demonstrated as a means to pole piezoelectric barium titanate nanofibers/nanowires. ${ }^{49,50}$

\section{Conclusion}

In this work, we have demonstrated a method for obtaining aligned barium titanate nanofibers using a rotating copper wire drum collector, where the alignment was maintained during calcination by one of two removal methods. We found and quantitatively tested a distribution model that appropriately fit the measured diameters of the barium titanate nanofibers. Additionally, we presented links between processing conditions and phase evolution in electrospun barium titanate nanofibers. By increasing the calcination time and temperature, the formation of a tetragonal barium titanate phase was promoted, leading to the formation of barium titanate nanofibers with enhanced piezoelectric properties. The use of a rotating drum collector, alongside optimized calcination conditions hold potential for the largescale synthesis of electrospun ceramic nanofibers with enhanced properties, greatly increasing their ability to be incorporated into novel devices and applications.

\section{References}

${ }^{1}$ J. H. Wendorff, S. Agarwal, and A. Greiner, Electrospinning: Materials, Processing, and Applications. Wiley-VCH Verlag GmbH \& Co. KGaA, Weinheim, Germany, 2012

${ }^{2}$ A. Koski, K. Yim, and S. Shivkumar, "Effect of Molecular Weight on Fibrous PVA Produced by Electrospinning," Mater. Lett., 58 [3-4] 493-7 (2004)

${ }^{3}$ P. Gupta, C. Elkins, T. E. Long, and G. L. Wilkes, "Electrospinning of Linear Homopolymers of Poly(Methyl Methacrylate): Exploring Relationships Between Fiber Formation, Viscosity, Molecular Weight and Concentration in a Good Solvent," Polymer (Guildf), 46 [13] 4799-810 (2005).

${ }^{4}$ P. Katta, M. Alessandro, R. D. Ramsier, and G. G. Chase, "Continuous Electrospinning of Aligned Polymer Nanofibers Onto a Wire Drum Collector," Nano Lett., 4 [11] 2215-8 (2004).

${ }^{5}$ Y. Dai, W. Liu, E. Formo, Y. Sun, and Y. Xia, "Ceramic Nanofibers Fabricated by Electrospinning and Their Applications in Catalysis, Environmental Science, and Energy Technology," Polym. Adv. Technol., 22 [3] 326-38 (2011)

${ }^{6}$ D. Li and Y. Xia, "Direct Fabrication of Composite and Ceramic Hollow Nanofibers by Electrospinning," Nano Lett., 4 [5] 933-8 (2004).

${ }^{7}$ A. Baji, Y.-W. Mai, Q. Li, S.-C. Wong, Y. Liu, and Q. W. Yao, "OneDimensional Multiferroic Bismuth Ferrite Fibers Obtained by Electrospinning Techniques.," Nanotechnology, 22 [23] 235702 (2011).

${ }^{8}$ D. Li and Y. Xia, "Fabrication of Titania Nanofibers by Electrospinning," Nano Lett., 3 [4] 555-60 (2003).

${ }^{9}$ K. Roh, D. C. Martin, and J. Lahann, "Biphasic Janus Particles With Nanoscale Anisotropy.," Nat. Mater., 4 [10] 759-63 (2005).

${ }^{10} \mathrm{P}$. Gupta and G. L. Wilkes, "Some Investigations on the Fiber Formation by Utilizing a Side-by-Side Bicomponent Electrospinning Approach," Polymer (Guildf), 44 [20] 6353-9 (2003).

${ }^{11}$ J. D. Starr and J. S. Andrew, "Janus-Type bi-Phasic Functional Nanofibers.," Chem. Commun. (Camb.), 49 [39] 4151-3 (2013).

${ }^{12}$ J. S. Andrew, J. D. Starr, and M. A. K. Budi, "Prospects for Nanostructured Multiferroic Composite Materials," Scr. Mater., 74 [3] 8-43 (2014)

${ }^{13}$ D. Li, Y. Wang, Y. Xia, and V. Uni, "Electrospinning of Polymeric and Ceramic Nanofibers as Uniaxially Aligned Arrays," Nano Lett., 3 [8] 1167-71 (2003).

${ }^{14}$ J. T. McCann, J. I. L. Chen, D. Li, Z.-G. Ye, and Y. Xia, "Electrospinning of Polycrystalline Barium Titanate Nanofibers With Controllable Morphology and Alignment," Chem. Phys. Lett., 424 [1-3] 162-6 (2006).

${ }^{15} \mathrm{X}$. Zhu and Z. Meng, "Operational Principle, Fabrication and Displacement Characteristics of a Functionally Gradient Piezoelectric Ceramic Actuator,"Sens. Actuators A Phys., 48, 169-76 (1995).

${ }^{16}$ K. K. Shung, J. M. Cannata, and Q. F. Zhou, "Piezoelectric Materials for High Frequency Medical Imaging Applications: A Review," J. Electroceramics, 19 [1] 141-7 (2007). 
${ }^{17}$ W. P. Mason and R. F. Wick, "A Barium Titanate Transducer Capable of Large Motion at an Ultrasonic Frequency," J. Acoust. Soc. Am., 23 [2] 209-14 (1951).

${ }^{18}$ L. S. Chen, S. L. Fu, and K. D. Huang, "Barium Titanate-Based Capacitors Buried Into Ceramic Substrates," Jpn. J. Appl. Phys. Part 2 Lett., 37 [10 Suppl B] L1241-3 (1998).

${ }^{19}$ J. Yuh, J. C. Nino, and W. M. Sigmund, "Synthesis of Barium Titanate (BaTiO3) Nanofibers via Electrospinning," Mater. Lett., 59 [28] 3645-7 (2005).

${ }^{20}$ K. Mimura, M. Moriya, W. Sakamoto, and T. Yogo, "Synthesis of BaTiO3 Nanoparticle/Poly(2-Hydroxyethyl Methacrylate) Hybrid Nanofibers via Electrospinning," Compos. Sci. Technol., 70 [3] 492-7 (2010).

${ }^{21}$ Y. He, T. Zhang, W. Zheng, R. Wang, X. Liu, et al., "Humidity Sensing Properties of BaTiO3 Nanofiber Prepared via Electrospinning," Sensor. Actuat. B Chem., 146 [1] 98-102 (2010).

${ }^{22}$ L. Wang, Y. He, J. Hu, Q. Qi, and T. Zhang, "DC Humidity Sensing Properties of BaTiO3 Nanofiber Sensors With Different Electrode Materials," Sensor. Actuat. B Chem., 153 [2] 460-4 (2011).

${ }^{23}$ J. D. Starr and J. S. Andrew, "A Route to Synthesize Multifunctional triPhasic Nanofibers," J. Mater. Chem. C, 1 [14] 2529-33 (2013).

${ }^{24}$ T. Benaglia, D. Chauveau, D. Hunter, and D. Young, "Mixtools: An R Package for Analyzing Finite Mixture Models. Journal of Statistical Software," J. Stat. Softw., 32 [6] 1-29 (2009).

${ }^{25}$ A. L. Yarin, W. Kataphinan, and D. H. Reneker, "Branching in Electrospinning of Nanofibers," J. Appl. Phys., 98 [6] 064501 (2005).

${ }^{26} \mathrm{~J}$. Malašauskiene and R. Milašius, "Mathematical Analysis of the Diameter Distribution of Electrospun Nanofibres," Fibres Text. East. Eur, 83 [6] 45-8 (2010).

${ }^{27}$ A. G. Gevorkyan, G. E. Shter, Y. Shmueli, A. Buk, R. Meir, and G. S. Grader, "Branching Effect and Morphology Control in Electrospun PbZr0.52Ti0.48O3 Nanofibers," J. Mater. Res., 29 [16] 1721-9 (2014).

${ }^{28}$ J. Deitzel, J. Kleinmeyer, D. Harris, and N. Beck Tan, "The Effect of Processing Variables on the Morphology of Electrospun Nanofibers and Textiles," Polymer (Guildf), 42 [1] 261-72 (2001).

${ }^{29}$ D. H. Reneker, A. L. Yarin, H. Fong, and S. Koombhongse, "Bending Instability of Electrically Charged Liquid Jets of Polymer Solutions in Electrospinning," J. Appl. Phys., 87 [9] 4531-47 (2000).

${ }^{30}$ R. T. Collins, J. J. Jones, M. T. Harris, and O. A. Basaran, "Electrohydrodynamic tip Streaming and Emission of Charged Drops From Liquid Cones," Nat. Phys., 4 [2] 149-54 (2008).

${ }^{31}$ A. Y. Malkin and A. I. Isayev, Rheology - Concepts, Methods, \& Applications. Chem Tec Publishing, Toronto, ON, Canada, 2006.

${ }^{32}$ F. J. Massey Jr, "The Kolmogorov-Smirnov Test for Goodness of Fit," J. Am. Stat. Assoc., 46 [253] 68-78 (1951).

${ }^{33}$ S. O'Brien, L. Brus, and C. B. Murray, "Synthesis of Monodisperse Nanoparticles of Barium Titanate: Toward a Generalized Strategy of Oxide Nanoparticle Synthesis," J. Am. Chem. Soc., 123 [48] 12085-6 (2001).

${ }^{34}$ P. K. Dutta and J. R. Gregg, "Hydrothermal Synthesis of Tetragonal Barium Titanate (BaTiO3)," Chem. Mater., 4 [4] 843-6 (1992).
${ }^{35} \mathrm{H}$. Xu, L. Gao, and J. Guo, "Preparation and Characterizations of Tetragonal Barium Titanate Powders by Hydrothermal Method," J. Eur. Ceram. Soc., 22 [7] 1163-70 (2002).

${ }^{36}$ M. Yashima, T. Hoshina, D. Ishimura, S. Kobayashi, W. Nakamura, et al., "Size Effect on the Crystal Structure of Barium Titanate Nanoparticles," J. Appl. Phys., 98 [1] 014313, 8pp (2005).

${ }^{37}$ E. Nyutu, C. Chen, P. Dutta, and S. L. Suib, "Effect of Microwave Frequency on Hydrothermal Synthesis of Nanocrystalline Tetragonal Barium Titanate," J. Phys. Chem. C, 112, 9659-67 (2008).

${ }^{38}$ T. Hoshina, H. Kakemoto, T. Tsurumi, S. Wada, and M. Yashima, "Size and Temperature Induced Phase Transition Behaviors of Barium Titanate Nanoparticles," J. Appl. Phys., 99 [2006] 1-9 (2006).

${ }^{39}$ L. Rimai, J. L. Parsons, J. T. Hickmott, and T. Nakamura, "Raman Spectrum of Long-Wavelength Phonons in Tetragonal Barium Titanate," Phys. Rev., 168 [2] 623-30 (1968).

${ }^{40}$ L. H. Robins, D. L. Kaiser, L. D. Rotter, P. K. Schenck, G. T. Stauf, and D. Rytz, "Investigation of the Structure of Barium Titanate Thin Films by Raman Spectroscopy," J. Appl. Phys., 76 [11] 7487-98 (1994).

${ }^{41}$ J. A. Sanjurjo, R. S. Katiyar, and S. P. S. Porto, "Temperature Dependence of Dipolar Modes in Ferroelectric $\mathrm{BaTiO}_{3}$ by Infrared Studies," Phys. Rev. B, 22 [5] 2396-403 (1980).

${ }^{42}$ M. P. Fontana and M. Lambert, "Linear Disorder and Temperature Dependence of Raman Scattering in $\mathrm{BaTiO}_{3}$," Solid State Commun., 10 [1] 1-4 (1972).

${ }^{43}$ V. Vinothini, P. Singh, and M. Balasubramanian, "Synthesis of Barium Titanate Nanopowder Using Polymeric Precursor Method," Ceram. Int., 32 [2] 99-103 (2006).

${ }^{44} \mathrm{Z}$. Chen and F. Peng, "Normal and Abnormal Grain Growths in $\mathrm{BaTiO}_{3}$ Fibers," J. Am. Ceram. Soc., 97 [9] 2755-61 (2014).

${ }^{45}$ Z. Zhou, H. Tang, and H. A. Sodano, "Vertically Aligned Arrays of BaTiO3 Nanowires," ACS Appl. Mater. Interfaces, 5 [22] 11894-9 (2013).

${ }^{46}$ Y. Zhuang, F. Li, G. Yang, Z. Xu, J. Li, et al., "Fabrication and Piezoelectric Property of $\mathrm{BaTiO}_{3}$ Nanofibers," J. Am. Ceram. Soc., 97, 2725-30 [JUNE] (2014).

${ }^{47}$ B. D. Huey, C. Ramanujan, M. Bobji, J. Blendell, G. White, et al., "The Importance of Distributed Loading and Cantilever Angle in Piezo-Force Microscopy," J. Electroceramics, 13 [1-3] 287-91 (2004).

${ }^{48}$ Z. Zhou, C. C. Bowland, M. H. Malakooti, H. Tang, and H. A. Sodano, "Lead-Free $0.5 \mathrm{Ba}\left(\mathrm{Zr}_{0.2} \mathrm{Ti}_{0.8}\right) \mathrm{O}_{3}-0.5\left(\mathrm{Ba}_{0.7} \mathrm{Ca}_{0.3}\right) \mathrm{TiO}_{3}$ Nanowires for Energy Harvesting," Nanoscale, 8 [9] 5098-105 (2016).

${ }^{49}$ C. Bowland, Z. Zhou, and H. A. Sodano, "Multifunctional Barium Titanate Coated Carbon Fibers," Adv. Funct. Mater., 24, 6303-8 (2014).

${ }^{50}$ C. C. Bowland, Z. Zhou, H. A. Sodano, C. C. Bowland, M. H. Malakooti, et al., "Scalable Synthesis of Morphotropic Phase Boundary Lead Zirconium Titanate Nanowires for Energy Harvesting," Nanoscale, 26 [45] 5098105 (2014). 